\title{
Author Index for Volume 100
}

Aaltonen, K 1055

Aaltonen, LA 511

Aavikko, M 1336

Abdi, E 1245

Abello, J 1755

Abnet, CC 551

Acevedo-Martinez, S 1073

Achen, MG 1784

Ackland, L 134

Adami, HO 1794

Adams, RA 251

Addou, S 1832

Afonso, L 487

Agarwal, R 305

Agarwal, S 941

Agostini, C 601

Ahmed, N 134

Ahn, JS 894

Aikou, T 153

Aithal, GP 178

Aittomäki, K 1055

Akhter, M 1812

Akiba, S 848

Al Olama, AA 426

Al Saati, T 1755

Alaiya, AA 1303

Alaoui-Jamali, MA 633

Al-Batran, SE 44

Aldaoud, A 44

Aleba, A 601

Alessandroni, P 881

Alexopoulou, DK 1659

Alfaro, C 1111

Alinger, B 1434

Allan, L 1867

Allen, NE 1817

Allum, W 1725

Almaguel, F 1073

Almeida, R 82

Alonzi, R 644

Altena, R 1861

Altés, A 1368

Altman, DG 1219

Aman, P 1687

Ambroisine, L 888

Ambros, IM 1471

Ambros, PF 1471

Ambrosone, CB 1680

Amini, R-M 1055

Amiot, M 366

Amoroso, D 1720

Amr, S 817

Anders, M 352

Andersen, CL 511

Anderson, AR 412

Anderson, JH 701, 1236

Anderson, LA 822

Anderson, LR 123

Anderson, M 1367

Anderson, N 923

Andersson, S 1303

Andersson, S-O 170
Ando, M 50

Andrèn, $\mathrm{O} 170$

Andreuccetti, M 1720

Andrews, EJ 1589

Andrews, L 583

Ang, J 1373

Ang, JE 671

Anton-Culver, H 412, 524

Antonini, NF 266

Antonuzzo, A 1720

Anttila, S 1336

Aoyagi, K 389

Apicella, C 583

Apolone, G 1566

Appleby, PN 1817

Arcusa, A 442

Ardanaz, E 1817

Ardern-Jones, A 376

Argilés, JM 311, 713, 1014, 1211

Arioka, H 50

Arkenau, H-T 1373

Arts, K 311, 713, 1014, 1211

Ashcroft, M 1515

Ashley, DM 1012

Ashley, S 305, 1373, 1725

Aspe, JR 1073

Asumen, MG 1073

Auer, G 1303

Auranen, A 1315

Autier, P 174

Azuara, D 1534

Azzato, EM 1806

Baba, H 1937

Baier, P 360

Baiget, M 1368

Baker, L 1687, 1867

Balanathan, P 1784

Balasubramanian, R 1879

Balbi, C 1608

Baldelli, AM 881

Baldwin, KA 1465

Ballarini, M 28

Balsitis, M 1530

Banerji, U 1373

Bang, Y-J 298, 732

Bangma, C 13

Banovich, F 259

Bapat, B 1966

Baracos, VE 1581

Baranov, V 1603

Barboro, P 1608

Barile, C 1549

Barker, CL 1558

Barlow, M 684

Barnadas, A 442, 1368

Barr, R 1026

Barrett-Lee, PJ 684

Barriuso, J 1373

Barros-Silva, JD 487

Barry, BD 1452

Barter, S 1873
Barthel, R 1755

Bassi, C 246

Bataille, R 366

Batra, S 1425

Baumgarten, M 817

Beau-Faller, M 985

Becker, S 1303

Beckers, J 656

Beesley, AH 1926

Beesley, J 412

Beiske, K 1627

Beith, J 1245, 1250

Belien, JAM 145

Bell, BA 789

Bell, D 1068

Bell, JL 96

Bellù, F 840

Belohlavek, C 322

Benlimame, N 633

Bennett, RL 1043

Benoit, G 608

Benson, M 1725

Bento, MJ 487

Beral, V 538

Berchuck, A 412

Berg, JP 450

Bergqvist, M 334

Bergstrom, S 334

Berkers, L-M 1240

Berndt, A 623

Berndt, SI 822

Berney, DM 888

Bernhard, H 44

Berns, EMJJ 971

Bernstein, L 524, 1483

Berridge, GLC 206

Berrino, F 1817

Berton, A 1444

Berton Rigaud, D 315

Bertrand, R 1896

Bertuccio, P 558

Bhalla, K 1523

Bhasin, D 106

Biankin, AV 123

Biasco, G 1017

Bibeau, F 1330

Bielawski, J 626

Bihrmann, K 1205

Bingham, S 1817

Birch, JM 188

Birgisson, H 1540

Birnbaum, D 1755

Bisonni, R 881

Bjartell, A 1540, 1799

Bjøro, T 455

Blaakaer, J 993

Blagden, S 707

Blanchard, F 1330

Blanks, RG 1043, 1832

Blomqvist, C 811, 1055

Blows, F 1806

Blum, HE 360
Boccardo, F 1608

Boccardo, S 1608

Bodman-Smith, M 1697

Boegl, M 1365

Boeing, H 1817

Bohlken, A 96

Boissière, F 1330

Bokaee, S 1889

Bolzonella, C 1549

Bonanomi, AG 259

Bonilla, M 1026

Boniol, M 174

Bonnard, C 1358

Bononi, A 1549

Bootle, D 315

Bordoni, A 1087

Bortoli, A 259

Bosch, FX 1191

Bosetti, C 558

Bougeard, G 1330

Bourbouloux, E 315

Bourré, L 723

Bouvier, V 1230

Boyd, AW 1095

Brandão, C 487

Brandi, G 1017

Brannan, RA 1393

Breakefield, XO 1603

Brenner, H 858

Brenton, JD 1873

Brewster, W 412

Brimmell, M 1347

Britton, P 1873

Brodeur, GM 1471

Brooks, A 707

Brouwer, CAJ 1861

Brown, G 1725

Brown, J 701

Brown, L 113

Brown, R 758

Brown, SBF 680, 807

Brundler, M-A 1292

Brunetti, IM 1720

Büchler, MW 246

Budroni, M 840

Bueno-de-Mesquita, HB 1817

Buffler, PA 863

Bugalho, MJ 1777

Bull, D 538

Bunch, KJ 213

Burchell, JM 1746

Burchill, SA 1627

Burger, H 228

Burger, M 1949

Burger, MPM 913

Burkart, C 44

Burniat, A 1013

Bursi, S 1720

Butt, AJ 123

Buxton, ILO 1465

Byrski, T 1508

Bziouech, H 1755 
Caldas, C 1517, 1873

Caldas, H 106

Calderan, L 1575

Calhau, C 1120

Callam, M 598

Calvo, A 932

Calvo, F 315

Cambien, B 1755

Cameron-Smith, D 134

Campana, F 1048

Campbell, C 1852

Campbell, FM 807

Campo, L 405

Campone, M 315

Camponovo, A 1087

Canaani, D 1213

Candiloro, I 405

Caniza, M 1026

Canney, PA 684

Cannon, SR 1406

Cantwell, MM 174, 1492

Cao, P 1267

Capellà, G 1534

Caporaso, NE 1806

Caraceni, A 1566

Card, TR 178

Cardwell, C 1492

Carew, JS 1523

Carlsen, NLT 853, 1212

Carmignani, G 1608

Carneiro, F 487

Carney, ME 412

Carter, PJ 1367

Casadomé, L 1534

Caspari, C 590

Castelao, JE 834

Castellsagué, X 1191

Castiglione, G 259

Catalano, V 881

Catena, R 932

Catney, D 174

Catusse, J 1949

Cavaco, BM 1777

Cazzola, L 259

Cejka, D 1739

Cen, L 106

Chamoto, K 1135

Chan, ATC 663

Chan, C 106

Chang, CJ 1765

Chang, ET 524

Chang, K-J 563

Chang, K-P 1002

Chang, M-C 1144

Chang, S-H 865

Chang, Y-S 1002

Chang-Claude, J 1680

Chaouachi, K 1015

Chapman, P 167

Chappell, E 1966

Charbonnier, F 1330

Chatenoud, L 558

Chau, I 1704, 1725

Chekerov, R 1731

Chen, C 412

Chen, C-A 1144

Chen, G-H 1002

Chen, H-C 1002
Chen, K-M 563

Chen, MF 1765

Chen, S 1912

Chen, S-J 1002

Chen, TH-H 563

Chen, X 412

Chen, Y-H 1002

Chen, Y-L 1144

Chenevix-Trench, G 412

Cheng, W-F 1144

Cheung, BB 96

Cheung, IY 1627

Chiang, Y-C 1144

Chiara, S 1720

Chirlaque, M-D 1817

Choe, K-J 732

Choi, DH 894

Choi, IS 298

Chomienne, C 918

Chong, PY 676

Chou, W-Y 578

Chou, Y-C 578

Choudhury, B 370

Christensen, JG 941

Christensen, LL 511

Christensen, S 1503

Christians, U 923

Christmann, M 322

Chu, C-H 578

Chua, S 305

Ciatto, S 259

Cid, MO 1777

Cisek, K 106

Clamp, AR 1

Clark, SJ 1534

Clarke, AR 221

Clarke, C 524

Clausen, H 1746

Clifford, G 532

Closset, J 1444

Clyne, M 376

Cnattingius, S 803

Coebergh, JWW 77, 901

Coffey, JC 1452

Cohn, SL 1471, 1627

Colditz, GA 611

Coleman, DA 1832

Coleman, LJ 1393

Coley, HM 571

Collins, DJ 644

Collins, TL 1755

Colomiere, M 134

Confortini, M 259

Contegno, F 28

Conti, DV 834

Conti, M 608

Cooke, TG 680, 807

Coombes, RC 160, 1061, 1879

Cooper, CS 240, 888

Copercini, M 1771

Copier, J 1697

Corey, E 1068

Corli, O 1566

Corrie, PG 1245

Cortés, A 1368

Cortessis, VK 834

Corvilain, B 1013

Cotter, TG 1452
Coumbos, A 1731

Coupland, VH 167, 527

Coyle, B 1292

Cozen, W 524

Craig, GT 1128

Cramer, DW 412

Cramer, T 772

Crea, P 123, 405

Crepaldi, G 1549

Cretin, J 601

Crippa, S 1087

Croci, D 28

Crook, T 571, 1687

Crozier, JEM 701

Culhane, AC 1452

Cunningham, D 1704, 1725

Cunningham, JC 412

Cupini, S 1720

Cutuli, B 1048

Cuzick, J 888

Cvancarova, M 455

Cybulski, C 1508

Czene, K 1358, 1486

Daducci, A 1575

Daftary, D 1966

Dahse, J 623

Dahse, R 623

Dai, M 532

Daidone, MG 739

Daito, M 494

Dal Maso, L 840

Dalgleish, A 1697

Daly, MB 583

Danjoh, I 389

Danos, O 1018

Darabi, H 1358

d'Arcy, JA 644

Darnton, A 1175

Darrington, RS 1165

Daskalow, K 772

Davidson, BR 617

Davidson, SE 1558

Davidson, WF 817

Davies, EA 167, 545

Davies, H 370

Davies, J 1889

Davies, M 1824

Davis, ID 1250

Day, JM 476

de Beaux, AC 63

de Bono, JS 671

De Dosso, S 1087

de Haas, EC 1861

de Kok, IMCM 1240

de Lafontan, B 1048

De Leon, M 1073

De Lisi, V 840

de Martel, C 194

de Pinieux, G 918

de Rosa, F 1017

de Sanjose, S 1191

de Souza, PL 649

De Vuyst, H 532

de Winton, E 693

de Wit, R 13

Deandrea, S 1566

DeAngelis, S 106
Deans, DAC 63

Deapen, D 524

Debniak, T 1508

De-Bono, J 1373

Decaestecker, C 1444

Decaudin, D 918

Degrassi, A 1575

del Río, E 1368

Demetter, P 1444

D'Emidio, S 881

Descamps, G 366

Devière, J 1444

Dewar, J 1867

Dhillon, A 617

Di Donato, S 1720

Di Fiore, F 1330

Diamandis, EP 1659

Diament, RH 1530

Diaz, M 1191

Diaz, R 932

Diaz Miqueli, A 950

Diaz-Gonzalez, JA 932

DiCioccio, RA 412, 993

Dieleman, LA 1581

Dietrich, P-Y 1771

Diez, S 1165

DiGiovanna, MP 941

Dilis, V 1817

Dilulio, J 37

Ding, J 494

Dinis-Ribeiro, M 487

Dirix, LY 971, 1277

Diss, T 1406

Ditsch, N 590

Dix, D 82

Dobrovic, A 1012

Doecke, JD 795, 1095, 1514

Doherty, JA 412, 993

Dohollou, N 601

Doi, R 246

Doki, Y 1647

Dolan, OM 174

Dollinger, MM 1032

Domingues, R 1777

Donato, A 840

Donnelly, D 174

Dono, K 1647

Donovan, J 426, 1198

Donovan, M 1452

dos Santos Silva, I 1824

Douard, R 608

Doughty, JC 680

Dowdy, S 89

Draper, GJ 213

Dreef, EJ 1617

Driemel, O 623

Driver, KE 1806

Droupy, S 608

Drummond, E 693

Drummond, KJ 1012

Duan, L 1483

Dubrot, J 1111

Dudek, AZ 1379

Duffy, SW 220, 1198, 1873

Duijm, LEM 77, 901

Dumont, J-E 1013

Duniho, S 1367

Durand, L 918 
Dutreix, C 315

Dvorak, AM 865

Dvorak, HF 865

Dyson, PJ 1061

Dzolganovski, B 82

Eastham, J 888

Easton, DF 426, 993

Ebert, M 352

Eckhardt, SG 923

Eden, TOB 188

Eder, W 1434

Edkins, S 370

Edlund, CK 412

Edwards, J 680, 807

Edwards, R 412

Edwards, S 426

Eeles, R 376, 426

Egashira, N 764

Eggleston, IM 723

Einarsdóttir, K 1358

Ekbom, A 1794

Eklund, CM 1846

Ekman, S 334

Eljamel, S 1867

Elsberger, B 807

El-Sheikh, S 959

Elst, HJ 1277

Enderling, H 1917

Endlicher, E 1032

Engebjerg, MC 200

Engels, EA 817, 822

Engelstaedter, V 360

Erro, L 1111

Eschwège, P 608

Eskens, FALM 228

Essink-Bot, M-L 70

Evans, WK 56

Eward, KL 959

Eyholzer, M 1343

Eyre, R 188

Faber, J 713, 1211

Falcini, F 840

Falcone, A 881, 1720

Fall, K 170

Falzon, M 959

Farace, P 1575

Farnell, DJJ 1558

Fay, M 693

Fearon, KCH 63

Fedeli, SL 881

Federico, M 840

Feichtinger, R 1434

Feltbower, RG 188

Fennelly, D 381

Ferrari, N 1608

Ferrazzi, E 1549

Ferreira, P 487

Ferrero, JM 601

Ferretti, S 840

Fichtner, I 950

Field, CJ 1581

Fietkau, R 291

Filipović, A 1879

Finch, A 421

Findlay, J 134

Finn, P 758
Firth, MJ 1926

Fisher, D 251

Fisher, G 888

Flaherty, KT 431

Flanagan, AM 1406

Fleuren, GJ 1617

Floor, K 145

Folini, M 739

Folprecht, G 44

Fondrinier, E 1048

Ford, J 1926

Forest, V 1896

Fornaro, L 881, 1720

Forouhi, P 1873

Fos, J 1343

Fosså, A 455

Fosså, SD 455

Foster, CS 240, 888

Foster, PA 476

Foulis, AK 701

Fourquet, A 1048

Fox, C 174

Fox, SB 405

Fracheboud, J 901

Fragoso, M 487

Franc, B 1013

Franceschi, S 194, 532, 840, 1191

Franklin, M 1379

Fraser, J 1315

Frattini, M 1087

Frebourg, T 1330

Frederiksen, K 185

Freedman, ND 551

Freitas, JR 1926

Fric, D 601

Friedman, HS 1012

Frigo, AC 1549

Friis, S 200, 1503

Frincke, J 1068

Fritzer-Szekeres, M 1739

Fuchs, M 1032

Fueger, BJ 1739

Fuentes, SL 1026

Fuereder, T 1739

Fuh, B 106

Fujiwara, Y 50

Fukagawa, T 153, 1937

Fukaya, M 389

Fung, MC 118

Fusco, M 840

Futreal, PA 370

Gäbele, E 1032

Gaber, A 1540

Gabra, H 707

Gadalla, S 822

Gadalla, SM 817

Gago-Dominguez, M 834

Gala, J-L 608

Galettis, PT 649

Galiè, M 1575

Galitovskiy, V 412

Galleges Ruiz, MI 145

Gallinger, S 1966

Galustian, C 1697

Gan, HK 1012

Gandellini, P 739

Gangadharan, P 848
Ganju, V 37

Gao, H 1957

García-Echeverría, C 1267

Garmo, H 170, 811

Garrett, C 1842

Garsd, A 1068

Gaskarth, M 1873

Gatter, KC 405

Gaub, MP 985

Gavin, AT 174

Gayther, SA 412, 993

Geay, J-F 601

Gedda, L 334

Geng, H 663

Gentry-Maharaj, A 412, 993

George, S 24

Gerald, W 888

Gerber, H-P 113

Ghaneh, P 246

Giaccone, G 145

Giacomin, A 840

Giard, S 1048

Gibbs, J 1842

Gietema, JA 1861

Gikas, P 1406

Gil, M 442

Giles, FJ 1523

Gilham, C 1175

Gilmour, IM 684

Giordani, P 881

Giovannetti, E 1120

Giuntini, F 723

Giustini, L 881

Glen, J 598

Glimelius, B 1540

Glynne-Jones, R 1666

Goedert, JJ 817

Goh, HK 663

Gohda, K 494

Goldhirsch, A 28

Gomez-Bougie, P 366

Gondos, A 858

Gonzalez, A 1111

Gonzalez, C 1817

González, S 442

Gonzalez-Moreno, O 932

Goode, EL 412

Goodman, MT 412

Goossens, A 913

Gopalan, A 888

Gordon, KA 113

Gores, GJ 1385

Gorselink, M 311, 713, 1014, 1211

Gorski, B 1508

Gorter, A 1617

Goto, M 344

Götze, H 908

Gøtzsche, PC 219

Gout, I 1406

Gouttebel, MC 601

Grabenbauer, GG 291

Graham, J 707

Grainge, MJ 178

Grande-Pulido, E 1111

Grant, RM 1867

Graziano, F 881

Grazzini, G 259
Greaves, M 863

Greco, MT 1566

Green, AC 795, 1514

Green, J 538

Greenberg, D 1198, 1806

Greenberg, ML 1026

Greenman, C 370

Greeno, EW 1379

Greenwood, CM 1966

Grell, K 185

Greten, TF 19

Gretschel, S 352

Grewal, IS 113

Griffiths, JR 789

Grillo, F 617

Groenewoud, JH 901

Gronwald, J 1508

Groshen, S 834

Grünberg, K 145

Grundy, RG 1292

Grunfeld, E 56

Guenther, T 1666

Guérin, E 985

Guittet, L 1230

Gullberg, B 1799

Gullbo, J 334

Gupta, S 1026

Gurpide, A 1111

Gusella, M 1549

Guy, M 426

Haagsman, HP 311, 1014

Haase, W 1680

Habbema, JDF 1103, 1240

Haber, M 96, 1471

Haddad-Guichard, Z 601

Hadjeres, R 1896

Hahnfeldt, P 1917

Hall, A 426

Hall, P 1358, 1486, 1794

Hall, PS 1513

Hallmans, G 1817

Hamdy, F 426, 1198

Hamel, E 932

Han, C 405

Han, S-W 298

Han, Y 1154

Hanby, AM 1393

Hansson, MG 8

Harada, H 747

Harder, J 360, 1032

Hardiman, P 1824

Hardy-Bessard, AC 601

Härkki, P 1315

Harrington, KJ 470, 1889

Harris, AL 405, 1666

Harris, LN 941

Hartmann, TN 1949

Harvey, R 979

Hasegawa, H 1943

Hasegawa, R 870

Hatch, J 1175

Hatmal, M 89

Hauss, J 908

Hawkins, CJ 1012

Hayashi, T 501

Haydon, A 1250

Haylock, RGE 206 
Hayward, O 1250

Hazelbag, S 1617

$\mathrm{He}, \mathrm{Q} 633$

Hedde, JP 291

Hedley, DW 1267

Heikkilä, P 1055

Helleman, J 971

Hellman, K 1303

Hellman, U 1303

Hellström, A-C 1303

Helmbold, I 1680

Hemminki, K 829, 1499

Henderson, K 524

Henshall, SM 123

Heo, DS 732

Herath, NI 1095

Heriot, AG 693

Hernandez-Suarez, G 1184

Hernes, E 450

Herrero, R 1191

Hersey, P 1250

Hervas-Stubbs, S 1111

Hesselius, P 334

Hestvik, UE 450

Hicks, RJ 693

Higashi, M 344

Higashino, F 1943

Higashiyama, S 1320

Higgins, DG 1452

Hiissa, J 1315

Hinke, A 1032

Hinz, A 908

Hirakawa, A 50

Hiraoka, M 747

Hirata, T 50

Hirata, Y 1320

Hirohashi, S 1257

Hirotsu, M 1957

Hiyama, E 1627

Hlatky, L 1917

Ho, YT 476

Höcker, M 352, 772

Hodgson, J 1175

Hoffmann, E 376

Hoffmann, M 44

Hogdall, CK 412, 993

Høgdall, E 412, 993

Hogg, A 693

Hol, L 1103

Hollenbeck, AR 551

Hollerbach, C 1032

Hollerbach, S 1032

Holmberg, L 811

Holt, C 959

Holt, SK 412

Holte, H 455

Honarpisheh, H 1393

Hoogerbrugge, N 266

Hopper, JL 583

Horgan, PG 701, 1236

Horlock, C 1061

Horsch, M 656

Horsman, D 421

Horvath, LG 1784

Hoshi, S-L 281

Hoskin, PJ 644

Hosoi, H 399

Hotakainen, K 1540
Houlston, RS 233, 1674

Houston, JG 1867

Howard, SC 1026

Howe, FA 789

Hrabé de Angelis, M 656

Hsieh, CC 1794

Hsieh, C-Y 1144

Hsieh, SY 1765

Hsu, G-C 578

$\mathrm{Hu}, \mathrm{Y}-\mathrm{H} 1144$

Huang, C-Y 1144

Huang, P 1912

Hubner, RA 233

Huebner, G 44

Huget, P 1277

Hughes, TA 1393

Huh, SJ 894

Huhtinen, K 1315

Humphreys, K 1358

Hunter, N 213

Hurme, M 1846

Hutzen, B 106

Huvila, J 1315

Huzarski, T 1508

Hyde, EI 1903

Hyland, J 381

Iacopetta, B 676

Idowu, B 1406

Iehara, T 399

Ikeda, M 870

Ikeda, S 1400

Iliadou, A 803

Im, S-A 298, 732

Im, Y-H 894

Imai, M 464

Inokuchi, M 782

Inoue, M 181

Intrieri, T 840

Introini, C 1608

Ishihara, H 494

Ishii, H 1937

Ishikawa, K 153

Ismail, M 1889

Isohata, N 389

Itasaka, S 747

Ito, S 1937

Ito, Y 676

Itoh, J 764

Iversen, ES 412

Iwasaki, M 181, 1812

Iwatsuki, M 1937

Iyer, R 1842

Jaakkola, PM 874

Jack, RH 545

Jacobs, IJ 993

Jaffey, J 56

Jahn, A 44

Jalkanen, J 1315

James, PW 188

Jansen, G 1120

Jansen, H 713, 1211

Jarrom, D 1903

Javle, M 1842

Jayalekshmi, PA 848

Jayatilleke, N 24

Jayson, GC 1
Jean-François, R 1896

Jenab, M 1817

Jenkinson, HC 188

Jennison, E 412

Jensen, AØ 200

Jensen, M-B 1205

Ji, J 829, 1499

Ji, SH 894

Jiang, X 834

Jin, L 1013

Jirström, K 1540

Joh, T 1320

Johansen, C 185

Johansson, J-E 170

Johansson, M 1540

Johns, TG 1012

Johnsen, NF 1817

Johnson, M 1837

Johnson, MG 1755

Johnston, S 305

Jonas, M 113, 1367

Jones, AL 274, 684

Jones, M 89

Jones, ND 1434

Jones, RL 305

Jöns, T 772

Jonsson, H 220

Jørgensen, KJ 219

Joseph, D 676

Josiah, D 106

Jöst, E 322

Judson, I 1373

Jugurnauth, S 426

Julien, S 1746

Junnila, J 1315

Kaaks, R 1817

Kahlert, S 590

Kaina, B 322

Kaiser, U 44

Kakiuchi, S 501

Kakuguchi, W 1943

Kamangar, F 551, 1016

Kamiya, T 1320

Kang, WK 894

Kaniwa, N 870

Kaplan, RS 251

Karagas, MR 200

Karimdjee, BF 1755

Karjalainen, A 1336

Kasai, S 1257

Kasamatsu, T 1400

Kataoka, H 1320

Kathmann, I 1120

Kato, T 1400

Katsumata, N 50

Kattan, MW 888

Kawano, T 782

Kawano, Y 1165

Kay, E 251

Kaye, AH 1012

Kaye, S 1373

Keane, M 1415

Keeney, GL 89

Kees, UR 1926

Kefford, R 1250

Kefford, RF 1245

Kegler, D 311, 713, 1014, 1211
Kelly, MA 1452

Kemmner, W 352, 772

Kendall, GM 213

Kenny, S 251

Kenter, GG 1617

Kerkmeijer, LG 979

Keshtgar, MR 1492

Kettunen, E 1336

Key, TJ 1817

Khamly, K 37

Khan, J 1471

Khan, S 1073

Khaw, K-T 1817

Khramtsov, A 1154

Killeen, SD 1589

Kim, D-W 732

Kim, J-S 732

Kim, M-A 298, 732

Kim, NK 732

Kim, SJ 494

Kim, SR 870

Kim, TM 732

Kim, T-Y 298, 732

Kim, WH 298, 732

Kim-Sing, C 421

King, M 274

Kingsbury, SR 959

Kirk, GD 799

Kirwan, WO 1452

Kiserud, CE 455

Kita, Y 153

Kitagawa, Y 1943

Kitamura, H 1135

Kitamura, T 1943

Kjaer, SK 412

Klaassen, RJ 82

Klassen, A 82

Klawitter, J 923, 923

Kneebone, R 24

Knight, JA 1966

Knops, AM 913

Ko, Y 291

Koach, J 96

Kobayashi, S 1647

Kocher, M 291

Koehm, J 590

Kofler, B 1434

Kohne, CH 44

Kojima, K 782

Kokubu, A 1257

Kolmakova, J 941

Komiya, S 1957

Kondo, M 281

Kondo, S 870

Konecny, GE 89

Koopman, M 266

Korangy, F 19

Korbelik, M 626

Korkeila, E 874

Kortman, GAM 266

Kosaka, Y 153

Kosmehl, H 623

Kotasek, D 1245

Kote-Jarai, Z 426

Kouno, T 50

Kratzke, RA 1379

Krauß, O 908

Kretzschmar, A 44 
Kromeyer-Hauschild, K 623

Kropp, S 1680

Krüger-Kjaer, S 993

Kruhøffer, M 511

Kubota, E 1320

Kudahetti, S 888

Kudo, E 501

Kuehn, W 1731

Kuhnt, S 908

Kuipers, EJ 70, 1103

Kujari, H 1315

Kulinskaya, E 160

Kullmann, F 44, 1032

Kumagai, J 782

Kumar, D 1697

Kumar, P 1379

Kumaran, GC 1

Kunitoh, H 464, 1037

Kuntner, C 1739

Kuo, W-H 563

Kuosma, E 1336

Kuppen, PJ 494

Kurahashi, N 181

Kuroda, Y 1438

Kuroshima, T 1943

Kurtz, DM 1385

Kusakabe, T 764

Kuvshinoff, B 1842

Kypta, RM 1165

Kyrgidis, A 670

La Vecchia, C 558

Lagiou, P 1794

Lamy, A 1330

Lancrenon, S 1048

Landgren, O 822

Langenberg, P 817

Langer, O 1739

Langridge, WHR 1073

Larranaga, N 1817

Larsson, H 853, 1212

Lassmann, S 360

Laudico, A 858

Launoy, G 1230

Laurberg, S 511

Lavaux, T 985

Laviano, A 311, 713, 1014, 1211

Law, C-L 113

Le Pessot, F 1330

Leathem, AJ 1492

Lecomte, N 918

Lee, $\mathrm{CH} 1765$

Lee, I-M 611

Lee, J-I 894

Lee, KH 298

Lee, K-U 732

Lee, K-W 298

Lee, MH 298

Lee, N-S 298

Lee, P-H 563

Lee, S-H 732

Leese, MP 476

Leggett, BA 1095

Legrain, M 985

Lehmann, A 908

Leibfritz, D 923

Leick, M 1949

Leitão, D 487
Leitch, EF 1236

Leite, V 1777

Leitzmann, MF 551

Lemanski, C 1048

Lemm, M 950

Lemos, C 1120

Lennartsson, J 334

Lennerz, V 322

Leong, T 37, 693

Leongamornlert, DA 426

Leppard, B 24

Lesniak, MS 1154

Lesniewski-Kmak, K 1379

Levack, P 1867

Levene, A 617

Levillain, R 1230

Lévy, E 601

Levy, V 315

Li, C 106

Li, L 1659

Li, L-K 532

Li, N 532

Li, P-K 106

Li, X 829

Li, Y 1358

Li, YQ 1358

Liao, W-L 1002

Lichtenegger, W 1731

Liebmann, A 908

Ligtenberg, MJL 266

Lilla, C 1680

Lim, DH 894

Limina, RM 840

Lin, J 106

Lin, L 106

Lin, YJ 1765

Lindblom, A 1674

Lindner, D 1287

Link, H 44

Linklater, KM 167

Linseisen, J 1817

Lipworth, L 1794

Lise, M 840

Little, P 24

Little, V 1824

Litwin, A 1842

Liu, B 532

Liu, C-Y 1002

Liu, ET 1358

Liu, J 1358

Lobitz, S 772

Loddo, M 959

Lomnytska, M 1303

London, WB 1471

Lopergolo, A 739

Lopes, C 487

Lopez, J 571

Lopez-Picazo, JM 1111

Loric, S 608

Lorigan, P 1250

Loubeyre, P 1771

Louie, KS 1191

Loupakis, F 881, 1720

Louwman, MWJ 77, 901

Lovejoy, DB 96

Lovric, MM 1012

Low, YL 1358

Lowe, J 1292
Lowy, A 24

Lu, Y 524

Lubinski, J 1508

Lucenteforte, E 558

Luiking, Y 713, 1211

Lundberg, F 803

Lurie, G 412

Lynge, E 220, 1205

Ma, H 524

MacKay, J 693

MacLean, AB 1824

MacRobert, AJ 723

Madeddu, A 840

Madi, A 251

Maeda, N 1438

Mæhlen, J 219

Maes, H 1277

Mahalingam, D 1415, 1523

Mahoney, CL 370

Maira, S-M 1267

Mäkinen, J 1315

Malek, NP 19

Malekzadeh, R 1016

Mallon, EA 680, 807

Mandelzweig, L 1021

Mangone, L 840

Manivet, P 608

Manno, M 1966

Manns, MP 19

Mantellini, P 259

Mapua, C 858

Marangoni, E 918

Maraveyas, A 1837

Marcuello, E 1368

Maréchal, R 1444

Margelí-Vila, M 442

Margison, GP 1245, 1250

Mari, D 881

Mariani, A 89

Marinelli, R 1549

Mariotte, N 1230

Maris, JM 1471

Marshall, C 598

Marshall, GM 96

Marsiglia, H 1048

Martin, RM 1198

Martin, V 1087

Martini, V 1755

Marubashi, S 1647

Marzola, P 1575

Mashima, T 1369

Masi, G 1720

Massard, G 985

Massuger, LF 979

Matera, A 37

Matsubara, K 1647

Matsumoto, K 50

Matsunoshita, Y 1957

Matsushima, T 494

Matsushita, M 1438

Matsuura, N 1647

Matthay, KK 1627

Matuszewski, M 1508

Maughan, TS 251

Maxwell, F 701

Maxwell, JA 1012

Maxwell, PH 1515
Maxwell, RJ 644

Mayer, D 1824

Mayor, R 1534

Mayr, JA 1434

Maziak, DE 56

Mazzucchelli, L 1087

McArthur, G 1250

McCabe, C 1513

McCarthy, O 274

McCormack, RT 160

McCormack, VA 1824

McDonald, PJ 1666

McElwaine, JN 1393

McGlynn, LM 807

McGown, G 1250

McGregor, JR 1530

McGuire, V 412, 993

McIntosh, HM 1852

McKee, RF 701, 1236

McKee, TA 1771

McKinney, PA 188

McLaughlin, JK 1503

McLeay, T 1867

McMillan, DC 701, 1236

McNally, RJQ 188

McNeil, CM 123, 405

McPherson, RAC 649

Meade, AM 251

Medina, JC 1755

Meerding, WJ 1240

Mehmet, H 1415

Mei, T 1912

Meijer, C 1184

Meijer, CJ 1191

Meijer, GA 145

Mekenkamp, L 266

Melero, I 1111

Méndez, F 1184

Menges, M 44

Menon, D 1549

Menon, U 412, 993

Merchant, S 626

Merigo, F 1575

Mery, E 1048

Messmann, H 1032

Metcalfe, KA 421

Meunier, A 1048

Meyer, B 1697

Meyer, T 1975

Michael, M 37

Michel, P 1330

Middleton, MR 1245, 1250

Middleton, RJ 174

Mikeska, T 1012

Miles, D 1746

Millar, EKA 123, 405

Miller, S 1292

Millet, MA 1755

Milner, AD 37, 693

Mimori, K 153, 1937

Mincheva-Nilsson, L 1603

Minn, $\mathrm{H} 874$

Minucci, S 28

Miot, F 1013

Mirasol-Lumague, MR 858

Mironenko, T 370

Misawa, A 399

Mitchell, H 707, 979 
Mizokami, M 181

Mizoshita, T 1320

Mizushima, T 1320

Molano, M 1184

Molinari, F 1087

Møller, H 167, 527, 545, 888

Monden, M 1647

Montanari, M 1566

Montero, E 950

Moore, M 246

Moore, PA 1367

Moorman, PG 412

Morash, C 56

Moreau, P 366

Moreno, A 442

Moreno, V 1184, 1534

Morey, AL 123

Morgan, A 436

Morgan, R 470

Mori, K 764

Mori, M 153, 1647, 1937

Mori, Y 1320

Morinibu, A 747

Morishita, M 1061

Morizane, C 870

Morrison, J 426

Mortimer, P 1245, 1250

Moss, SM 1043, 1832

Moug, SJ 1530

Moura, MM 1777

Moutereau, S 608

Moyes, LH 1236

Moysich, KB 412, 993

Mrkonjic, M 1966

Mubwandarikwa, E 188

Mucci, L 170

Muderspach, L 89

Mueller, BU 1343

Mueller, M 1739

Muir, K 426

Muirhead, CR 206, 213

Mulcahy, H 381

Mulholland, S 426

Muller, A 37

Muller, RP 291

Muñoz, Á 1184

Muñoz, M 442

Muñoz, N 1184, 1191

Munro, AJ 1867

Munzone, E 28

Murakami, M 1647

Murayama, T 782

Murillo, R 1184

Murray, LJ 174

Musgrove, EA 123

Nagano, H 1647

Nagy, JA 865

Nagy, N 1444

Nair, RRK 848

Nakagawara, A 1471

Nakanishi, H 1937

Nakasono, M 501

Nakayama, S 1438

Nam, DH 894

Nambooze, S 799

Nandi, S 1154

Nannuru, KC 1638
Narod, SA 421, 1508

Nasrollahzadeh, D 1016

Natsugoe, S 153

Naumann, SC 322

Nawrocki, ST 1523

Nazareth, I 274

Neal, DE 426, 1198

Neal, RD 1852

Neale, RE 795, 1514

Near, AM 412

Negri, E 558, 1566

Neoptolemos, JP 246

Ness, RB 412, 993

Nesterova, A 1367

Neuhaus, T 291

Neuhausen, SL 524

Neuper, C 89

Neureiter, D 1434

Neuville, A 985

Nevanlinna, H 1055

Newman, SP 476

Ng, M 693

Ng, SS 676

Ngan, SY 37, 693

Nguewa, PA 932

Nicolato, E 1575

Nieto, J 1824

Nilsson, G 811

Nilsson, J 1603

Nishida, Y 344

Nishimura, T 1135

Nishio, M 464

Nishiwaki, Y 464

Nizar, S 1697

Noda, K 464

Noda, T 1647

Noguchi, S 494

Nokihara, H 1037

Nolè, F 28

Nomoto, M 344

Nordstrand, A 1603

Norris, M 96

Northover, JMA 1666

Nöthlings, U 1817

Numada, S 494

Nuver, J 1861

Nymark, P 1336

Oates, J 1725

O’Brien, L 426

O’Brien, M 305

Ocama, P 799

Ochiya, T 1257

O’Connor, D 1347

Oden-Gangloff, A 1330

O’Donoghue, D 381

Odumosu, O 1073

Offman, J 376

Oflazoglu, E 113

Ogasawara, N 1320

Ogretmen, B 626

Oh, D-Y 298

O'Hagan, JA 206, 213

Ohe, Y 1037

Ohkuri, T 1135

Ohta, A 389

Ohta, H 389

Ojima, H 1257
Okines, A 1725

Okulicz, W 1794

Okusaka, T 870

Olesen, AB 200

Olmos, D 671, 1373

Olschewski, M 360

Olsen, AH 1817, 1205

Olsen, JH 1503

Olsson, H 1799

Onda, T 1400

O'Neill, A 1873

Opio, CK 799

Opitz, OG 360

Opstad, KS 789

Orfeuvre, H 601

Orlotti, NI 739

Ørntoft, TF 511

O’Rourke, K 56

Osamura, YR 764

Oskay-Oezcelik, G 1731

O'Sullivan, J 381

O’Toole, SA 123, 405

Otremba, B 44

Otto, F 360

Oudet, P 985

Overton, C 1824

Overvad, K 1817

Owen, E 246

Pabst, T 1343

Packham, G 1347

Padbury, R 246

Padhani, AR 644

Padrini, R 1549

Palazón, A 1111

Palli, D 1817

Palmer, C 1245

Palmieri, C 160

Pamecha, V 617

Pande, A 1842

Pandey, ON 1379

Pandeya, N 795, 1514

Pandha, HS 470, 1889

Pantel, K 160

Paraiso, D 601

Paré, L 1368

Park, K 894

Park, MJ 894

Park, SR 298

Park, W 894

Park, YH 894

Parkes, S 188

Parsonnet, J 194

Pashayan, N 1198

Pasini, F 1549

Patel, D 56

Paterson-Brown, S 63

Payne, R 160

Peacock, SJ 583

Pearce, CL 412, 993

Pearce, MS 188

Pearson, ADJ 1471, 1627

Pedersen, BL 853

Pedersen, JS 1784

Peh, BK 676

Peinado, MA 1534

Pelicci, PG 28

Pelucchi, C 558
Penault-Llorca, F 1048

Pennati, M 739

Percharde, M 571

Perera, KU 1926

Perez, R 950

Perez-Gracia, JL 111

Perheentupa, A 1315

Pesenti, E 1575

Peter, MB 1393

Peters, GJ 145, 1120

Petersen, BL 1212

Pethe, VV 1966

Peto, J 1175

Petrini, I 1720

Pezzella, F 405

Pfeiffer, M 1949

Pfeiffer, RM 817, 822

Pharoah, P 1198

Pharoah, PDP 412, 993, 1806

Phelan III, J 1842

Phesse, TJ 221

Phillipson, MA 206, 213

Picco, G 1746

Pierce, JS 626

Piesiak, W 1508

Piffer, S 840

Pike, MC 834, 993

Pinese, M 123

Pinto, AE 1777

Pioud-Martigny, R 1048

Pirianov, G 1415

Pirie, K 538

Pirinen, R 1336

Piselli, P 840

Pitfield, T 160

Plowright, L 470

Plumb, JA 758

Plummer, CJ 684

Plummer, M 194, 532

Pockney, P 24

Podratz, KC 89

Polder, JJ 1240

Polesel, J 840

Polinder, S 70

Poll, A 421

Polus, M 1444

Poma, P 739

Ponder, BAJ 993

Pontén, F 1540

Poon, FF 663

Popanda, O 1680

Posso, H 1184

Postel-Vinay, S 1373

Postma, A 1861

Potter, BVL 476

Poulsen, AH 1503

Poupon, M-F 918

Poutanen, M 1315

Praud, D 558

Presneau, N 1406

Press, MF 89

Preusser, M 1739

Prevost, T 959

Price, T 37

Prieto, L 442

Primrose, J 24

Prinz, C 1365

Pritchard, S 82 
Proctor, I 959

Pross, M 352

Provencal, J 601

Pujade-Lauraine, É 601

Pulte, D 858

Punt, CJA 266

Puppo, P 1608

Purdie, C 1687

Purohit, A 476

Putter, H 494

Pyrhönen, S 874

Qian, ZR 501

Qiao, Y-L 532

Qiu, G-H 663

Quaye, L 993

Quinlan, P 1687

Quoix, E 985

Radovanovic, I 1020

Radulovic, M 376

Raghavan, D 1287

Rahman, MM 501

Rajan, B 848

Rake, C 1175

Ramus, SJ 412, 993

Ranson, M 1245, 1250

Rasheed, S 1666

Rashid, M 959

Rasul, S 1879

Rautenberg, TA 1513

Ray-Coquard, I 601

Raymond, E 315

Reading, C 1068

Rebolj, M 1240

Rechnitzer, C 853, 1212

Recktenwald, CV 656

Redaniel, MT 858

Redmond, HP 1452, 1589

Redrado, M 932

Reed, MJ 476

Reeves, GK 538

Regan, N 106

Rêgo, S 487

Reijerink, JCIY 1103

Repaci, E 1608

Reuter, V 888

Reynolds, P 524

Rha, SY 663

Ribeiro, R 1026

Riboli, E 1817

Ricci, S 1720

Richard-Fiardo, P 1755

Richardson, A 274

Richardson, DR 96

Riddell, A 1725

Riehle, D 89

Riethdorf, S 160

Riffkin, CD 1012

Riley, C 134

Riley, RD 1219

Rimm, DL 941

Rinaldi, S 1817

Risbridger, GP 1784

Robb, SD 684

Roberts, LR 1385

Robinson, D 527

Robsahm, TE 450
Rocca, A 28

Röcken, C 352

Roddam, AW 1817

Rodriguez, JA 145

Rogers, HA 1292

Rogers, M 274

Rohwer, N 772

Rolff, J 950

Ronderos, M 1184

Roos, WP 322

Ros, MM 1817

Rose, P 1852

Rosenberg, L 1486

Ross, JA 63

Rosset, A 1771

Rossi, D 881

Rossi, M 558

Rossing, MA 412, 993

Rosthoej, S 853, 1212

Routledge, JA 1558

Roxburgh, CSD 701

Roy, S 1128

Royer, R 421

Rubagotti, A 1608

Rubeca, T 259

Ruben, SM 1367

Rumjahn, SM 1465

Ruosaari, S 1336

Ruppert, A-M 985

Russo, A 840

Ryan, MC 1367

Sabharwal, A 1250

Sabourin, J-C 1330

Sacerdote, C 1817

Sadanandam, A 1638

Sadeghi, S 1514

Sadetzki, S 1021

Sadhegi, S 795

Saeki, N 389

Sahu, RP 1425

Saijo, N 464, 870

Sainsbury, R 959

Saito, Y 870

Sakamoto, H 389

Salazar, J 1368

Saldanha, JD 1530

Saletti, P 1087

Salmaggi, A 28

Salmenkivi, K 1336

Salmon, I 1444

Salto-Tellez, M 676

Salvat, J 601

Salvi, S 1608

Samali, A 1415

Samoli, E 1794

Sánchez, M-J 1817

Sano, T 501

Santarius, T 370

Santos, JR 1777

Santos, L 89, 487

Sasajima, Y 1400

Sasaki, H 389, 1957

Sasaki, M 1320

Sasako, M 153, 1937

Sasazuki, S 1812

Sauerbrei, W 1219

Saunders, MI 644
Sautter-Bihl, M-L 1680

Savage, P 1061

Sawada, J 870

Sawada, M 1400

Sawyer, MB 1581

Sbarbati, A 1575

Scardino, P 888

Schädler, S 656

Schaedel, D 1731

Schaeffner, E 360

Schairer, C 817

Schatzkin, A 551

Schedvins, K 1303

Schepeler, T 511

Schildkraut, JM 412

Schlag, PM 352, 772

Schleiermacher, G 1471

Schleutker, J 1846

Schmezer, P 1680

Schmidt, CW 322

Schmidt, LS 185

Schmid-Alliana, A 1755

Schmid-Antomarchi, H 1755

Schmiegelow, K 185

Schmitt, F 487

Schmitt-Graeff, A 1949

Schoenleber, SJ 1385

Schoffski, P 44

Schroeder, H 853, 1212

Schueller, H 291

Schüz, J 185

Schwarz, R 908

Schwarz, S 623

Scoazec, JY 1755

Scorilas, A 1659

Scott, J 421

Searle, PF 1903

Seckl, MJ 979

Sedano, L 1368

Seeger, RC 1627

Segara, D 405

Sehgal, A 167

Sehouli, J 1731

Seimiya, H 1369

Sekine, I 1037

Sekyere, E 96

Seliger, B 656

Sellers, TA 412

Selwood, DL 1209

Semba, S 1438

Separovic, D 626

Serkova, NJ 923

Serraino, D 840

Serrano, D 932

Setälä, M 1315

Setoguchi, T 1957

Sewell, R 1746

Shaaban, AM 1393

Shah, K 1191

Shah, M 1806

Shah, N 676

Shah, R 1687

Shalaby, A 1406

Shand, N 315

Shanley, S 376

Sharma, R 707

Sheahan, K 381

Shehadeh, NJ 1379
Sheridan, J 381

Sherris, D 932

Shi, J-F 532

Shibata, D 834

Shibata, T 464, 1257

Shibayama, M 494

Shiels, MS 799

Shimizu, C 50

Shimoda, T 389

Shimura, T 1320

Shindoh, M 1943

Shinomiya, K 747

Shirane, M 764

Shu, X 829, 1499

Sier, CF 1617

Siersema, PD 70

Silva, RR 881

Silver, A 1666

Simon, R 511

Singer, S 908

Singh, RK 1638

Singh, S 1638

Sinnatamby, R 1873

Sinnett, HD 160

Skog, J 1603

Skoglund Lundin, J 1674

Slade, MJ 160, 1879

Sleijfer, DTh 1861

Slimani, N 1817

Smadja-Joffe, F 918

Smalley, KSM 431

Smit, AJ 1861

Smit, EF 145

Smit, VTHBM 494

Smith, D 246

Smith, H 24

Smith, IE 305

Smith, L 1393

Smith, LM 1367

Smith, MJ 1452

Smith, P 1687

Smith, S 96

Sobo, M 106

Soerjomataram, I 77

Solano, S 1111

Solaymani-Dodaran, M 178

Sonabend, AM 1154

Song, B 1674

Song, H 412, 993

Song, HS 298

Sooman, L 334

Soong, R 676

Soosaipillai, A 1659

Sørensen, FB 511

Sørensen, HT 200, 1503

Spanevello, MD 1095

Speight, PM 1128

Speirs, V 1393

Speleman, F 1471

Spencer, EA 1817

Sperl, W 1434

Spina, B 1608

Spitale, A 1087

Spitz, R 1471

Spurdle, AB 412

Srivastava, SK 1425

Stacker, SA 1784

Stahl, M 44 
Stalpers, LJA 913

Starling, N 1725

Stassen, LPS 70

Stattin, P 1817

Stavridi, F 1725

Stebbing, J 160, 1061

Steele, N 758

Steinbach, S 44

Steinberg, SM 145

Steinberg, W 1303

Stenman, U-H 1540

Stern, DF 941

Stevens, C 370

Steyerberg, EW 70

Stickney, D 1068

Stier, S 291

Stievano, L 1549

Stirling, JJ 644

Stocken, DD 246

Stoeber, K 959

Stone, IJ 113

Stott, B 1061

Stracci, F 840

Stratton, MR 370

Strickland, AH 37

Strommer, S 1739

Su, J 633

Suárez, LR 1817

Suarez, N 1111

Suchy, J 1508

Sugihara, K 782

Sugimoto, T 399

Sugiyama, E 870

Suligoi, B 840

Sullivan, TJ 1755

Sullivan-Halley, J 524, 1483

Sultana, R 501

Sun, C-A 578

Sun, P 421, 1508

Sundquist, J 829, 1499

Sundquist, K 829, 1499

Sundström, J 874

Sung, JJY 663

Sung, L 82, 1026

Sutherland, MK 1367

Sutherland, RL 123, 405

Sutton, A 436

Suvitie, P 1315

Swanton, C 1517

Sweep, FC 979

Swerdlow, AJ 1832

Swindell, R 1558

Swords, R 1523

Syrjänen, K 874

Szegezdi, E 1415

Szwiec, M 1508

Tagliabue, G 840

Takagi, Y 782

Takano, E 405

Takeda, Y 1647

Takekoshi, S 764

Takemasa, I 1647

Talbot, IC 1666

Talieri, M 1659

Talvinen, K 874

Talwalkar, JA 1385

Tammela, TLJ 1846
Tamminen, A 1055

Tamura, K 50

Tamura, T 464, 1037

Tan, BH 63

Tan, EY 405

Tanai, C 1037

Tanaka, M 1957

Tanaka, S 399, 1943

Tanaka, Y 181

Tang, HL 118

Tang, HM 118

Tani, T 389

Tanida, S 1320

Taniguchi, H 389

Tao, Q 663

Tarca, AL 626

Tarp, M 1746

Taylor, NJ 644

Taylor-Papadimitriou, J 1746

Teall, TJ 1393

Teixeira, MR 487

Tekkis, PP 1666

Terent, A 811

Terry, KL 412

Tessandori, R 840

Testori, A 28

Thai, J 1465

Thomas, B 89

Thomas, CM 979

Thomas, J 1725

Thomas, NPB 1245

Thomas, RJ 598

Thomas, W 96

Thompson, AM 1687

Thompson, J 1725

Thomsen, HF 200

Thomsen, RW 1503

Thomson, D 1250

Thorncroft, M 1250

Thorsen, K 511

Thunnissen, FBJM 145

Tian, L 663

Tichet, J 1230

Tilanus, HW 70

Tinge, B 334

Tirabosco, R 1406

Titus-Ernstoff, L 412

Tjalma, WAA 971

Togashi, Y 1135

Toi, M 281

Tokuda, Y 764

Tong, L 1912

Tookman, A 274

Torresi, U 881

Torres-Rendon, A 1128

Tosetto, M 381

Toso, S 1549

Tosti, G 28

Totsuka, Y 1943

Tougeron, D 1330

Tovey, SM 680

Tran, KTC 70

Trauger, R 1068

Travis, RC 1817

Trenerry, MK 134

Tretli, S 450

Triaridis, S 670
Trichopoulos, D 1794, 1817

Trichopoulou, A 1817

Trinh, XB 971

Troisi, R 1794

Trojan, J 1032

Truini, M 1608

Tsau, H-S 563

Tsuchiya, K 399

Tsuda, H 1400

Tsugane, S 181, 1812

Tsuji, M 848

Tsuruo, T 1369

Tsutsumida, H 344

Tucker, K 583

Tudur Smith, C 246

Tuech, J-J 1330

Tumino, R 840, 1817

Tunon de Lara, C 1048

Turley, H 405, 1666

Tusquets, I 442

Tutill, HJ 476

Tymrakiewitcz, M 426

Ubbink, DT 913

Ueno, $\mathrm{H} 870$

Ulasov, IV 1154

Umemura, S 764

Umeshita, K 1647

Untch, M 590

Urquhart, R 56

Ursin, G 524

Usadel, H 360

Uysal-Onganer, P 1165

Vallee, JP 1230

van Alphen, RJ 228

van Ballegooijen, M 1103, 1240

van Dam, PA 971, 1277

van de Poll-Franse, LV 901

van de Velde, CJH 494

Van Den Berg, DJ 412, 993

van den Berg, MP 1861

Van den Eynden, G 971

Van der Auwera, I 971, 1277

van der Beek, EM 311, 713,

$$
1014,1211
$$

van der Gaast, A 70

van der Togt, ACM 1103

van der Valk, H 1103

van Doorn, L-J 194

van Haaften, G 370

van Helvoort, A 311, 713, 1014, 1211

van Krieken, JHJM 266

Van Laere, SJ 971, 1277

Van Laethem, J-L 1444

van Leerdam, ME 1103

Van Marck, EA 1277

van Nes, JGH 494

van Norren, K 311, 713, 1014 1211

Van Orden, KL 1367

van Rooijen, N 113

van Tuijl, S 311, 1014

van Vuuren, AJ 1103

van Weerden, WM 13
Vandrovcova, J 1674

Vanhala, E 1336

Varbanova, M 360

Varney, ML 1638

Vasile, E 1720

Vass, SO 1903

Vejborg, I 1205

Velentzis, LS 1492

Venot, C 366

Vercelli, M 840

Vercoutter-Edouart, A-S 1746

Verghese, ET 1393

Vermeulen, PB 971, 1277

Verrill, MW 684

Verschuur, EML 70

Verspaget, HW 511

Vieira, J 487

Vierkant, RA 412

Vieth, M 352, 772

Vincent, TJ 213

Virgo, JD 376

Visioli, CB 259

Vitarelli, S 840

Vitonis, AF 412

Vodermaier, A 590

Voegeli, A-C 985

von Fournier, D 1680

Wabinga, HR 799

Wacheck, V 1739

Wacher, J 853, 1212

Wada, H 1647

Wada, T 1320

Wailoo, A 436

Wakita, D 1135

Wall, NR 1073

Wallis, MG 1873

Wallström, P 1799

Walsh, G 305

Wanders, A 334

Wanek, T 1739

Wang, EL 501

Wang, H 1784

Wang, H-J 89

Wang, J 563, 1912

Wang, JH 1452, 1589

Wang, L-M 381

Wang, X-H 1347

Wang, Y 376, 1912

Ward, AC 134

Ward, B 160

Wardega, P 334

Wardley, AM 684

Wark, PA 1824

Watanabe, K 464

Watson, AJ 1245, 1250

Watson, E 1852

Watters, JM 56

Waxman, J 1165

Webb, A 571

Webb, PM 412, 795, 1514

Weber, B 601

Wedrén, S 1358

Weller, D 1852

Weller, RE 1926

Wenz, F 1680

Werelius, B 1674

Werner, M 360 
Werzowa, J 1739

West, D 524

West, J 178

White, S 1068

Whiteman, DC 795, 1514

Whittemore, AS 412, 993

Widmark, A 1603

Wiedenmann, B 352, 772

Wiemer, EAC 228

Wigmore, SJ 63

Wihlm, J-M 985

Wikman, H 1336

Wilkens, LR 412

Wilkinson, C 1852

Wilkinson, R 426

Willcock, T 206

Williams, ED 1784

Williams, GH 959

Williams, M 598

Williams, R 274

Wilschut, JA 1103

Wilson, JI 644

Wilson, M 723

Wilson, WR 1903

Winterhoff, B 89

Wirfält, E 1799

Wishart, GC 1873

Wokolowczyk, D 1508

Wolin, KY 611
Wong, AHY 663

Woodside, JV 1492

Woodward, N 617

Wotherspoon, A 1725

Wozniak, E 993

Wu, AH 412, 993, 1483

Wu, L 1912

Wu, M-H 578

Wu, R-F 532

$\mathrm{Wu}, \mathrm{X} 334$

Xie, X 747

Xu, A 1912

$\mathrm{Xu}, \mathrm{B} 1794$

$\mathrm{Xu}, \mathrm{Y} 633$

Xue, H 1581

Xynopoulos, D 1659

Yagüe, E 1879

Yagyu, S 399

Yamada, H 782

Yamada, N 344

Yamaji, T 1812

Yamamoto, N 1037

Yamamoto, S 1037

Yamanaka, Y 50

Yan, M 405

Yan, Y 611
Yang, G 1912

Yang, H-K 732

Yanofsky, R 82

Ychou, M 1330

Yeh, CN 1765

Yen, AM-F 563

Yi, SY 894

Ying, J 663

Yokdang, N 1465

Yokobori, T 1937

Yokoyama, A 464

Yokozaki, H 1438

Yonemori, K 50

Yonezawa, S 344

Yoshida, T 389, 870

Yoshikawa, D 1257

Yoshioka, S 1647

Young, A 864

Young, J 959

$\mathrm{Yu}, \mathrm{C}-\mathrm{P} 578$

Yu, J 663, 1842

Yu, J-C 578

$\mathrm{Yu}, \mathrm{VPCC} 376$

Yuan, J-M 834

Yuen, KL 118

Yunokawa, M 50

Zadeh, G 1020

Zaffaroni, N 739
Zahl, P-H 219

Zaidi, SAA 160

Zalcberg, JR 37

Zambon, P 840

Zanetti, R 840

Zanke, BW 1966

Zappa, M 259

Zelada-Hedman, M 1674

Zeng, L 747

Zeps, N 676

Zerillo, C 941

Zhang, W 206, 213

Zhang, W-H 532

Zhang, Y-Z 532

Zhao, G 1912

Zhao, Y 1912

Zheng, Y 1659

Zhou, X 1674

Zhu, L 1912

Zhu, Y 747

Zijlmans, HJ 1617

Zimmermann, FA 1434

Ziogas, A 412

Zoellner, U 315

Zorzi, M 259

Zsebedics, M 1739 